MATEC Web of Conferences 1, 05007 (2012)

DOI: $10.1051 /$ matecconf $/ 20120105007$

(C) Owned by the authors, published by EDP Sciences, 2012

\title{
The Nonlinear Tuned Vibration Absorber
}

\author{
R. Viguié and G. Kerschen,a \\ Space Structures and Systems Laboratory, Dept. of Aerospace and Mechanical Engineering, University of Liège, Belgium
}

\begin{abstract}
The objective of this paper is to introduce a new nonlinear dynamical absorber, the nonlinear tuned vibration absorber, through a rigorous nonlinear extension of the tuning rule for the linear tuned vibration absorber. This nonlinear tuning methodology combined with the increased suppression bandwidth brought by the intentional use of nonlinearity leads to the development of an absorber that is effective in wide ranges of frequencies and motion amplitudes. The results are illustrated using a one-degree-of-freedom primary system.
\end{abstract}

\section{Introduction}

Controlling nonlinear dynamical instabilities represents a great challenge, because nonlinear systems exhibit complex phenomena, which linear systems cannot. Specifically, the frequency of nonlinear oscillations depends intrinsically on motion amplitude. Any anti-vibration device that ignores this nonlinear property simply does not work. Unfortunately, this statement holds for the linear tuned vibration absorber (TVA), which is tuned to a specific problematic resonance frequency.

A handful of American laboratories [1-3] have proposed the constructive utilization of nonlinearity for vibration absorption. For instance, the nonlinear energy sink, an essentially nonlinear absorber, can extract energy from any mode of a host structure, a result that has never been obtained with linear absorbers [4]. This unconventional way of thinking renders the state of affairs in nonlinear dynamics particularly exciting, as pointed out in [5].

Although nonlinear absorbers can absorb disturbances in wide ranges of frequencies due to their increased bandwidth, their performance depends critically on motion amplitude [5]. One important observation is that all previously developed nonlinear absorbers are designed without considering the frequency-energy dependence of the nonlinear oscillations of the host structure. Typically, an absorber with polynomial nonlinearity is considered (e.g., quadratic [1] or cubic [3]), regardless of the nonlinear phenomena at the source of the instabilities.

The objective of this paper is to develop the nonlinear tuned vibration absorber (NLTVA), a new passive device for the effective and robust suppression of nonlinear instability.

\section{The free response case}

The linear TVA is the most widely used device for vibration control in engineering. Its mass and stiffness are chosen so that its resonance frequency matches that of a specific problematic resonance frequency of the host structure. This condition is necessary for vigorous energy ex-

\footnotetext{
${ }^{a}$ e-mail: g.kerschen@ulg.ac.be
}

changes between the primary structure and the attachment; it is clearly at the basis of the success of the TVA.

In view of the frequency-energy dependence of nonlinear oscillations, the proposed nonlinear absorber relies on a rigorous nonlinear extension of this linear tuning rule. The tuning will ensure that the NLTVA resonance frequency matches that of the considered nonlinear instability, and this for all motion amplitudes of interest. This tuning condition combined with the increased suppression bandwidth brought by the intentional use of nonlinearity leads to the development of an absorber that is effective in wide ranges of frequencies and motion amplitudes.

The results reported in reference [6] shows that the functional form of the absorber nonlinearity should be such that the NLTVA backbone curve possesses the same dependence on energy as that of the primary system. For instance, if a purely cubic primary oscillator is considered, then the absorber nonlinearity should also be purely cubic. The equations of motion of the coupled system are:

$$
\begin{aligned}
m_{1} \ddot{x}_{1}+c_{1} \dot{x}_{1}+c_{2}\left(\dot{x}_{1}-\dot{x}_{2}\right)+k_{n l_{1}} x_{1}^{3}+k_{n l_{2}}\left(x_{1}-x_{2}\right)^{3} & =0 \\
m_{2} \ddot{x}_{2}+c_{2}\left(\dot{x}_{2}-\dot{x}_{1}\right)+k_{n l_{2}}\left(x_{2}-x_{1}\right)^{3} & =0(1)
\end{aligned}
$$

The primary oscillator is impulsively loaded. The parameters of the primary system are $m_{1}=1 \mathrm{~kg}, m_{2}=0.05 \mathrm{~kg}$, $k_{n l_{2}}=0.0025 \mathrm{~N} / \mathrm{m}^{3}$, and $c_{1}=c_{2}=0.002 \mathrm{Ns} / \mathrm{m}$. Figure 1 represents the percentage of impulsive energy eventually dissipated in the light-weight nonlinear absorber. When $k_{n l_{1}}$ is in the range [0.1-0.35] $\mathrm{N} / \mathrm{m}^{3}$, the NLTVA dissipates $90 \%$ of the energy for all impulse values considered. This dynamics is similar to that of a linear TVA coupled to a linear primary oscillator.

\section{The forced response case}

The forced system is now considered

$$
\begin{aligned}
& m_{1} \ddot{x}_{1}+c_{1} \dot{x}_{1}+c_{2}\left(\dot{x}_{1}-\dot{x}_{2}\right)+ \\
& \quad k_{n l_{1}} x_{1}^{3}+k_{n l_{2}}\left(x_{1}-x_{2}\right)^{3}=F \cos \omega t \\
& m_{2} \ddot{x}_{2}+c_{2}\left(\dot{x}_{2}-\dot{x}_{1}\right)+k_{n l_{2}}\left(x_{2}-x_{1}\right)^{3}=0
\end{aligned}
$$

with $F=0.1 \mathrm{~N}$ and $m_{1}=1 \mathrm{~kg}, m_{2}=0.05 \mathrm{~kg}, k_{n l_{1}}=1 \mathrm{~N} / \mathrm{m}^{3}$, $k_{n l_{2}}=0.0025 \mathrm{~N} / \mathrm{m}^{3}$, and $c_{1}=c_{2}=0.002 \mathrm{Ns} / \mathrm{m}$. Its fre- 


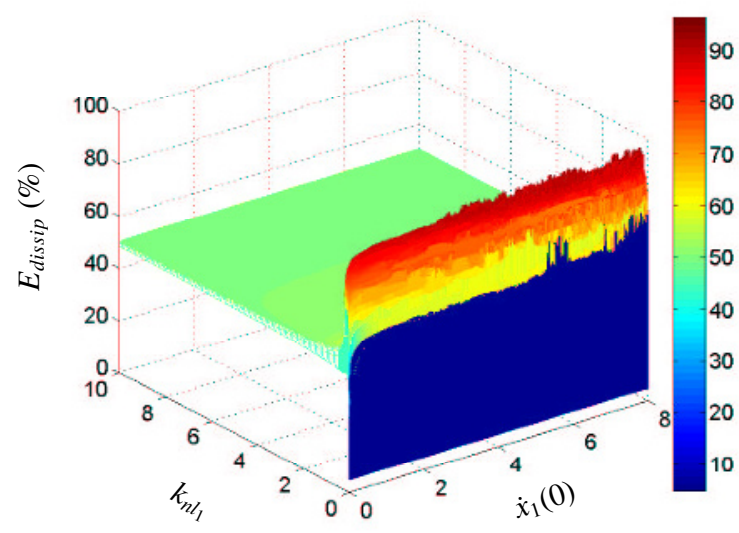

Fig. 1. Energy dissipated in the NLTVA against the nonlinear stiffness of the primary system and the impulse magnitude.

quency response (NLFRF) is computed using the MATCONT software and is represented in Figure 2(b). The frequency response of the uncontrolled case (i.e., without the nonlinear absorber) is depicted in Figure 2(a).

The design of the NLTVA, i.e., the determination of appropriation values for $k_{n l_{2}}$ and $c_{2}$ is carried out using bifurcation analysis. Indeed, Figure 2(b) illustrates that the points of maximum amplitude $\alpha, \beta$ and $\gamma$ correspond to bifurcations (limit points, LP). Tracking such bifurcation in parameter space $\left(k_{n l_{2}}, c_{2}\right)$ is therefore a possible means of optimizing the NLTVA performance. However, because the excitation frequency is also a necessary parameter during numerical continuation, a codimension three continuation problem is to be solved, which is beyond the current capability of MATCONT. The solution is to optimize the absorber performance with respect to the nonlinear stiffness, which is a codimension two problem. The same procedure can then be carried out for damping, and the whole process is repeated until convergence.

The continuation of bifurcations $\alpha, \beta$ and $\gamma$ with respect to $k_{n l_{2}}$ and $\omega$ is shown in Figure 3. The first interesting feature is that the bifurcation point $\gamma$ is eliminated for $k_{n l_{2}}>0.007601 \mathrm{~N} / \mathrm{m}^{3}$. At point $R_{1}$, the locus of bifurcation $\gamma$ meets the locus of another bifurcation point (different from $\alpha$ and $\beta$ ), which eventually leads to the elimination of the two bifurcations. In addition, for $k_{n l_{2}}=0.0076515$ $N / m^{3}$ denoted by $\theta$, the two remaining bifurcations $\alpha$ and $\beta$ are such that the corresponding resonance peaks have the same amplitude, which is the selected tuning condition in the forced case; the TVA is also designed such that the two FRF peaks have the same amplitude. The peak amplitude is reduced by approximately $50 \%$ after this first iteration.

The optimization of the damping coefficient is the next step of the procedure. The initial guess is the point computed by the previous iteration, i.e., $k_{n l_{2}}=0.0076515\left[\mathrm{~N} / \mathrm{m}^{3}\right]$ and $c_{2}=0.002[\mathrm{Ns} / \mathrm{m}]$. The details of this procedure are explained in [7]. The sequential optimization of the absorber nonlinear stiffness and damping coefficients can be continued, and convergence is reached after only 3 iterations. Eventually, the peak amplitude is reduced by $85 \%$.

Table 1 compares the results for three different forcing amplitudes $F$. Substantial peak reduction can be obtained in all three cases, which highlights the excellent performance of the NLTVA. It also turns out that, while the

\begin{tabular}{cccccc}
\hline Set & Force & Iteration & $k_{n l_{2}}$ & $c_{2}$ & $\%$ of $X_{1}$ reduction \\
\hline 1 & 0.01 & 3 & 0.0104 & 0.0079 & 78 \\
2 & 0.1 & 3 & 0.0103 & 0.0170 & 85 \\
3 & 1 & 3 & 0.0103 & 0.0365 & 90
\end{tabular}

Table 1. Optimal absorber configuration for different forcing amplitudes $F$.

nonlinear stiffness seems to be intrinsic to the system, the choice of damping is to be made according to the tradeoff between performance (i.e., peak reduction maximization) and robustness (i.e., sensitivity to forcing amplitude). Small damping results in lower performance, but the absorber is effective in a larger range of forcing amplitudes. Conversely, large damping results in increased performance, but the absorber is effective in a narrower range of forcing amplitudes. Interestingly, the same trade-off exists for the TVA, but, for this linear absorber, the performance is to be balanced against the frequency range of interest.

The same procedure was applied to a more general primary oscillator comprising both a linear spring and a cubic spring. In this case, the NLTVA also possesses linear and cubic springs. Peak amplitude reduction of at least $85 \%$ was also achieved for all considered excitation levels [7].

\section{Conclusion}

The objective of this paper is to develop an absorber which can mitigate the vibrations of a nonlinear system in a wide range of input energies. The backbone of the absorber should possess the same dependence on energy as that of the backbone of the primary system. To fulfill this frequency-energybased tuning condition, the functional form of the absorber nonlinearity is to be carefully selected.

\section{References}

1. B. Hall, D.T. Mook, A.H. Nayfeh, S. Preidikman, A Novel Strategy for Suppressing the Flutter Oscillations of Aircraft Wings, AIAA J. 39 (2001), 1843-1850.

2. S.W. Shaw, P.M. Schmitz, A.G. Haddow, Dynamics of Tautochronic Pendulum Vibration Absorbers: Theory and Experiment, J. of Comp. and Nonl. Dyn. 1 (2006), 283-293.

3. A.F. Vakakis, O.V. Gendelman, Energy Pumping in Nonlinear Oscillators, J. of Appl. Mech. 68 (2001), 4248.

4. G. Kerschen, J.J. Kowtko, D.M. McFarland, L.A. Bergman, A.F. Vakakis, Theoretical and Experimental Study of Multimodal Targeted Energy Transfer in a System of Coupled Oscillators, Nonl. Dyn. 47 (2007), 285309.

5. S. W. Shaw, B. Balachandran, A Review of Nonlinear Dynamics of Mechanical Systems in Year 2008, J. of Syst. Design and Dyn. 2 (2008), 611-640.

6. R. Viguié, G. Kerschen, Nonlinear Vibration Absorber coupled to a Nonlinear Primary System: a Tuning Methodology, J. of Sound and Vib. 326 (2009), 780-793.

7. R. Viguié, Tuning Methodology of Nonlinear Vibration Absorbers coupled to Nonlinear Mechanical Systems, PhD Thesis, Univ. of Liège, 2010. 
CSNDD 2012
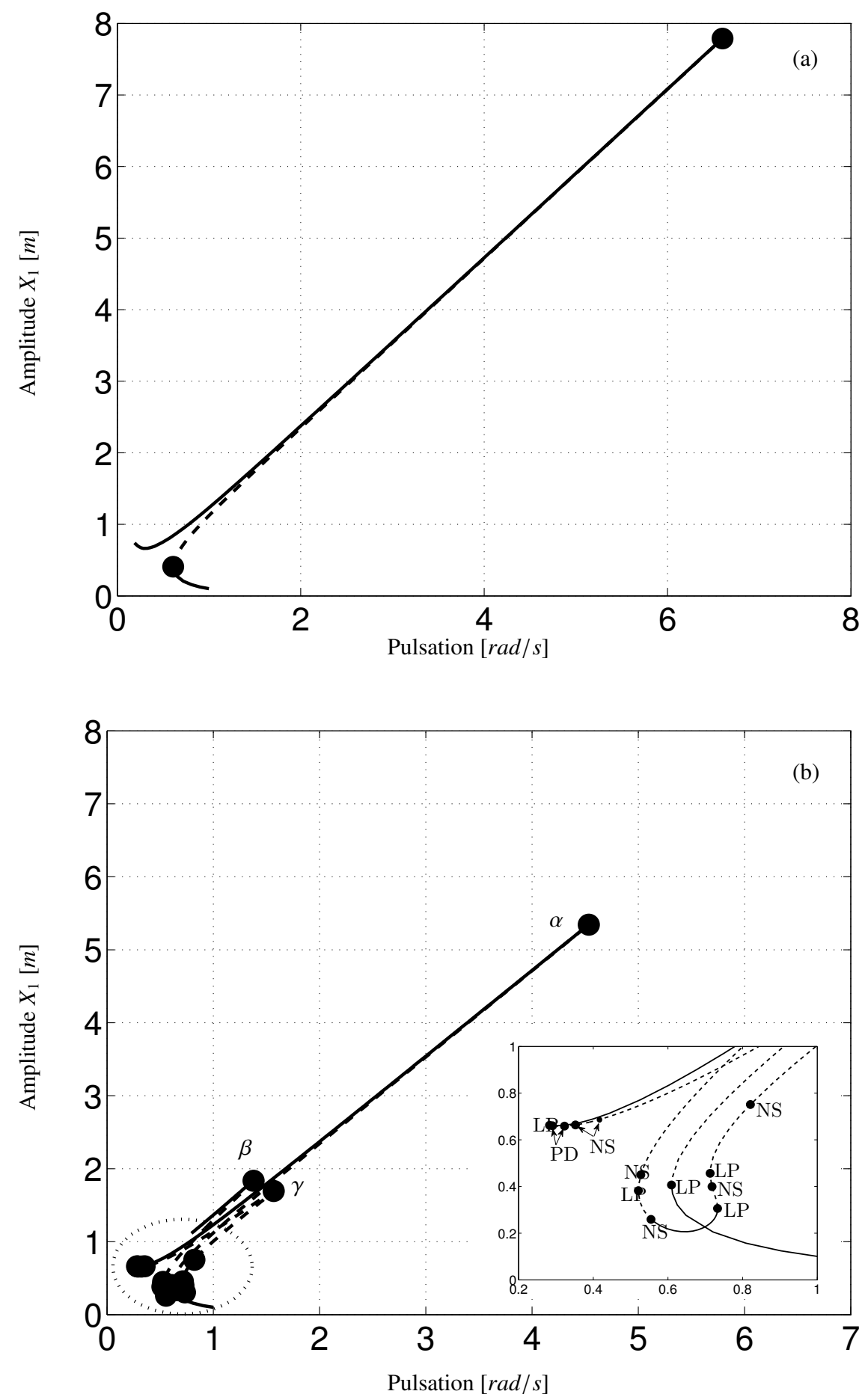

Fig. 2. Frequency response for $\mathrm{F}=0.1 \mathrm{~N}$. (a) Uncontrolled response; (b) controlled response with $k_{n l_{2}}=0.0025 \mathrm{~N} / \mathrm{m}^{3}$. Solid and dashed lines correspond to stable and unstable periodic solutions, respectively; dots are related to bifurcation points. 


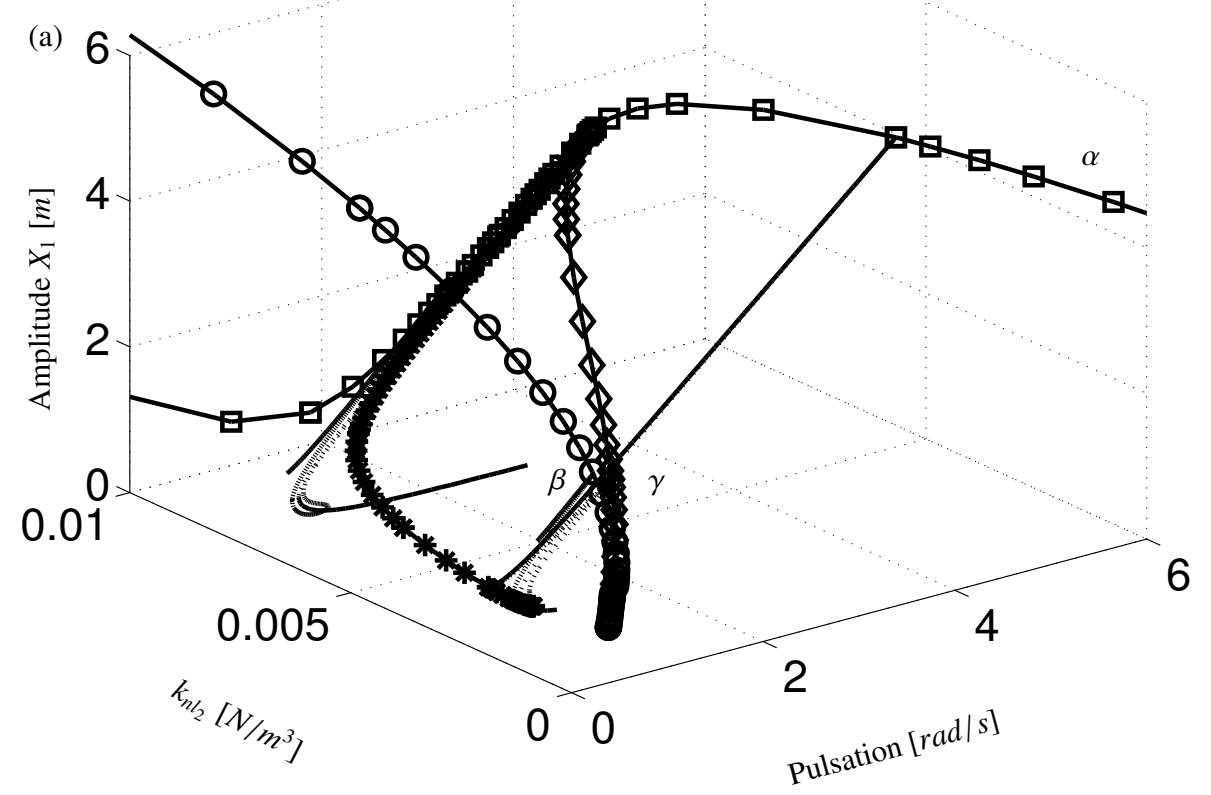

(b)

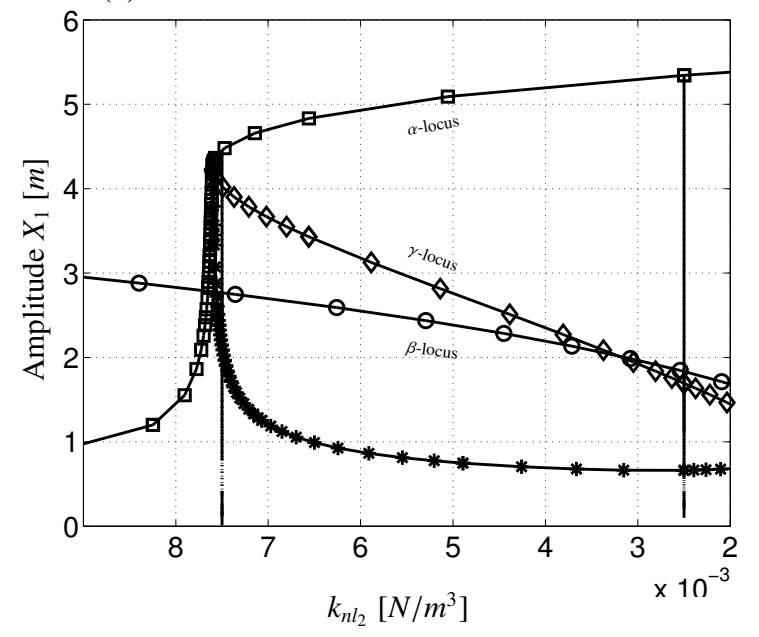

(c)

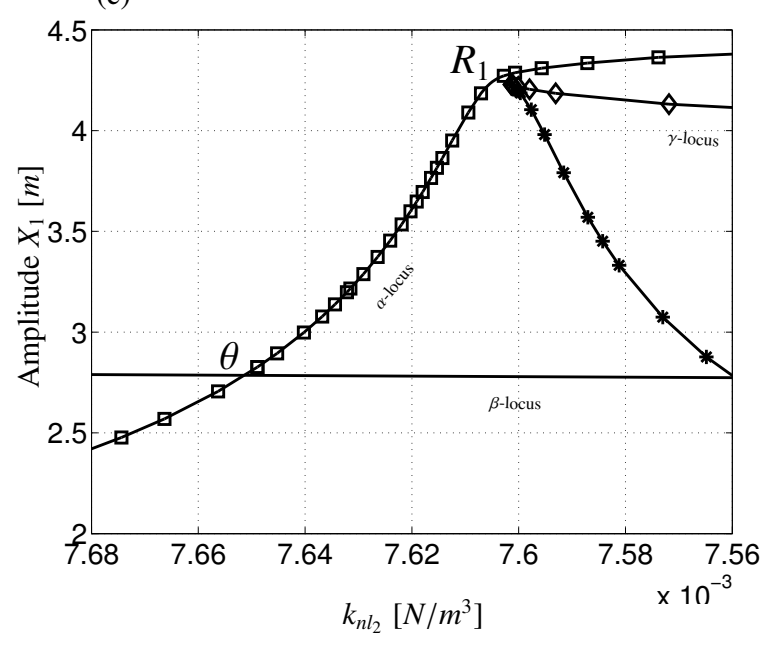

Fig. 3. Bifurcation tracking versus $k_{n l_{2}}$. Solid and dotted lines without symbols are related to the stable and unstable parts of the NLFRFs, respectively. Solid lines with squares, circles and diamonds are related to bifurcation loci. (a) Three dimensional plot; (b) two-dimensional projection in the plane $\left(k_{n l_{2}}, X_{1}\right)$; (c) close-up of the $2 \mathrm{D}$ projection around the optimal region. 